\title{
Quantitative Estimation of Free Volume Distribution of Polymers with Photochromic Reactions
}

\author{
Mitsue Sato and Takashi Yamashita \\ Department of Pure and Applied Chemistry, Faculty of Science and Engineering, Tokyo \\ University of Science, 2641 Yamazaki, Noda 278-8501, Chiba, Japan

\begin{abstract}
Reactions in polymer solid are different from those in solutions because the mobility of polymer molecules is so restricted that reactivities vary in the local environment and site by site. One of the factors influencing on their reactivity is free volume of the polymer, however free volume has not been quantitatively estimated up to now. In this paper, azobenzene in polymethyl methacrylate was used as photochromic probe. Azobenzene in PMMA were isomerized with photoirradiation and the change in the absorption spectra of azobenzene was observed. Quantum yield distribution for the photoreaction was obtained by analyzing the observed curve of isomerization, and the effect of annealing temperature on their quantum yield distribution is also described.
\end{abstract}

Keywords: free volume distribution, solid state polymer, quantum yied, photoisomerization, azobenzene.

\section{Introduction}

Many organic photofunctional materials, such as PHB, optical memory, and photoresist use the reactions in polymer solids. In order to develop such polymer materials, it is necessary to analyze their microstructure or nanostructure because the reactions in solid state polymers are often affected by their solid state structures, and they are generally different from those in solutions.

Photochemical and photochromic reactions are often utilized as a key step of the reactions in a variety of functional materials. They are also used as probes to investigate microstructure of various polymers. We have reported on free volume of polymers with photochromic probe technique [1], fluorescent probe technique [2], photochemical hole burning [3], positron annihilation techniques [4-6], where the free volume of polymers explains the inhomogeneity of the polymers qualitatively. Recently we have analytically solved the differential equation of a photochromic reaction, and reported the way to analyze photochemical reactions quantitatively [7]. One of the most important feature of solid state reactions in polymers is the existence of the distribution of various kinetics due to the inhomogeneity of the matrix However there have been no reports on the distribution of the kinetics using photoreactive probes. In this paper, we first clarify the distribution of kinetics of solid state reactions in polymers by deconvolution of the kinetics of a photochromic reaction in polymer solid, and then describe the free volume distribution of polymers.

\section{Method}

\subsection{Sample Preparation}

Azobenzene was purchased from Tokyo Kasei Kogyo Co., and was purified by recrystallization from ethanol. Methyl methacrylate (MMA, Tokyo Kasei Kogyo Co.) was distilled under vacuum before use.

A $0.1 \mathrm{~mol} / / \alpha, \alpha$-azobisisobutyronitrile (Tokyo Kasei Kogyo Co.) and azobenzene were dissolved in MMA, then the mixture was heated at $50^{\circ} \mathrm{C}$ for 14 hours to polymerize.

The prepared films were annealed by heating at $80^{\circ} \mathrm{C}$ or $100^{\circ} \mathrm{C}$ for 1 hour in an oven, then they were cooled to room temperature overnight.

\subsection{Measurements of Photoisomerization in Solution}

Solutions of azobenzene in ethanol were photoirradiated by $317,325,350$ and $372 \mathrm{~nm}$ lights of a Xenon lamp (USHIO UBH300), and the absorption spectra of the samples were measured by a UV detector (Jasco V-550). Actinometry was carried out with a digital power meter (ADVANTEST TQ8210). 


\subsection{Measurements of Photoisomerization in PMMA Film}

Absorbance of PMMA films containing azobenzene at irradiation wavelength was measured by the UV photometer, irradiating $325 \mathrm{~mm}$ or $350 \mathrm{~mm}$ light in the same way.

\section{Results and Discussion}

\subsection{Photoisomerization of Azobenzene in Solution}

Figure 1 shows absorption spectrum change of azobenzene in ethanol solution during photoirradiation of $317 \mathrm{~nm}$ light. As the absorption band of trans-azobenzene at $317 \mathrm{~nm}$ was decreased, a new absorption of cis form appeared at $240 \mathrm{~nm}$ and $440 \mathrm{~nm}$, which showed photoisomerization from trans-form to cis-form occurred. Isosbestic points at $236 \mathrm{~nm}, 268 \mathrm{~nm}$ and $372 \mathrm{~nm}$ were also observed.

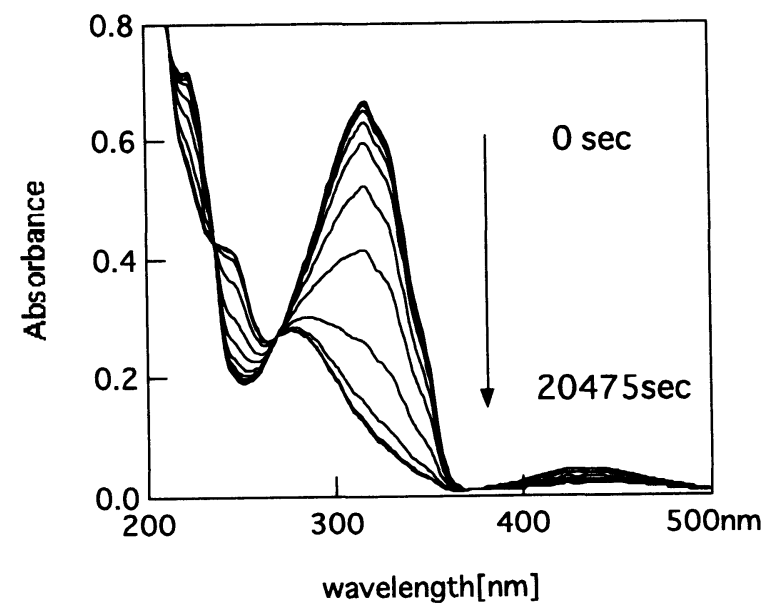

Figure 1. The UV absorption spectra of azobenzene in ethanol solution during photoirradiation of $317 \mathrm{~nm}$ light at photoirradiation times of $0,15,35,75,155,315,635,1275$, $2555,5115,10235$, and 20475 seconds.

As to the photoreaction, the change in the total number of the photoreactive molecules in a unit time, is proportional to the product of the total number of absorbed photons in a unit time and quantum yield of the photoreaction, given in Eq.1,

$$
-\frac{d\left(10^{-3} C S l\right)}{d t}=I_{0} S\left(1-10^{-6 l}\right) \phi
$$

where $C\left[\mathrm{~mol} \mathrm{l}^{-1}\right]$ is the concentration of the photoreactive molecules, $S\left[\mathrm{~cm}^{2}\right]$ is the sample area, $l$ $[\mathrm{cm}]$ is the sample thickneess, $I_{0}$ [einstein $\mathrm{cm}^{-2} \mathrm{~s}^{-1}$ ] is the incident light intensity, $\varepsilon\left[\mathrm{M}^{-1} \mathrm{~cm}^{-1}\right]$ is the molar extinction coefficient of the sample at the photoirradiating wavelength, and $\phi$ is the quantum yield of the photoreaction of the molecule, respectively.

In the photochromic reaction such as present photoisomerization of azobenzene, however, the rate of the decrease in the trans concentration is given as the difference between the reactions from trans to cis and from cis to trans photoisomerization. Therefore the rate equation for the photochromic reaction is expressed as Eq.2,

$$
\begin{aligned}
& -\frac{d\left(10^{-3} C_{\text {trans }} S l\right)}{d t} \\
& =I_{0} S \frac{\varepsilon_{\text {trans }} C_{\text {trans }}}{\varepsilon_{\text {trans }} C_{\text {trans }}+\varepsilon_{c i s} C_{c i s}}\left(1-10^{-O D}\right) \phi_{\text {trans } \rightarrow \text { cis }} \\
& -I_{0} S \frac{\varepsilon_{\text {cis }} C_{c i s}}{\varepsilon_{\text {trans }} C_{\text {trans }}+\varepsilon_{c i s} C_{c i s}}\left(1-10^{-O D}\right) \phi_{\text {cis } \rightarrow \text { trans }}
\end{aligned}
$$

where $C_{\text {trons }}$ and $C_{c a s}\left[\mathrm{~mol} \mathrm{l}^{-1}\right]$ are the concentration of trans- and cis-forms, $\varepsilon_{\text {rras }}$ and $\varepsilon_{\text {cis }}\left[\mathrm{M}^{-1} \mathrm{~cm}^{-1}\right]$ are their molar extinction coefficients at the photoirradiating wavelength, and $\phi_{\text {rems } \rightarrow x \text { is }}$ and $\phi_{c i s-\text { trons }}$ are the quantum yields of the photoreaction, respectively.

Experimentally one can observe the optical density of the sample, i.e., total optical density of both trans and cis-forms. Hence Eq. 2 can be transformed into Eq.3,

$$
\begin{aligned}
\frac{d O D}{d t}= & 10^{3} I_{0}\left(\varepsilon_{\text {trans }} \phi_{\text {trans } \rightarrow \text { cis }}+\varepsilon_{\text {cis }} \phi_{\text {cis } \rightarrow \text { trans }}\right) \\
& \times \frac{1-10^{-O D}}{O D_{0}}\left(\frac{O D_{\infty}}{O D}-1\right)
\end{aligned}
$$

where $O D_{0}$ and $O D_{\infty}$ are the optical density at photoirradiation wavelength before photoirradiation and after photostationary state, respectively. The quantum yields for the photochromic reaction $\phi_{\text {mons } \rightarrow \text { is }} \phi_{c i s-t \text { roms }}$ should be known to understand the solid state reactions, and so we should know both $\varepsilon_{\text {mrons }}$ and $\varepsilon_{\text {cis }}$ to obtain the quantum yields. However, it is difficult to measure the $\varepsilon_{c i s}$ because cis-azobenzene is so unstable to isolate. So, we calculated the molar extinction coefficient of the cis form by an experiment photoirradiating either peak or isosbestic point described below.

Observed optical density at time $t[\mathrm{sec}]$ is the sum of those of trans and cis form during photoirradiation, given in Eq.4, based on the Lambert-Beer's law,

$$
\begin{aligned}
& O D_{o b s}(t) \\
& \quad=\varepsilon_{\text {trans }}(o b s) C_{\text {trans }}(t) l+\varepsilon_{c i s}(o b s) C_{c i s}(t) l
\end{aligned}
$$

where $O D_{o b s}(t)$ is the optical density of the sample at the wavelength of obs at time $t, C_{\text {trous }}(t)$ and $C_{c i s}(t)[\mathrm{mol} / \mathrm{l}]$ are the concentration of the photochromic molecules, 
$l[\mathrm{~cm}]$ is the sample thickness, respectively. The molar extinction coefficient $\varepsilon_{\text {trons }} \varepsilon_{c i s}$ are rewritten as $\varepsilon_{\text {mens }}(o b s)$, $\varepsilon_{c i s}(o b s)$, where obs is the wavelength of measurement. The equation can be applied to whole wavelength. Eq. 4 is rewritten as Eq. 5 after photostationary state.

$$
\frac{O D_{o b s}^{i r r}(\infty)}{O D_{o b s}^{i r r}(0)}-1=\left(\frac{\varepsilon_{c i s}(o b s)}{\varepsilon_{\text {trans }}(o b s)}-1\right) \frac{C_{c i s}^{i r r}(\infty)}{C_{\text {trrans }}^{i r r}(0)}
$$

where $O D s$ are the optical densities, $C s\left[\mathrm{~mol}^{-1}\right]$ are the concentrations of trans- or cis-form, and $\varepsilon_{\text {trons }}$ and $\varepsilon_{c i s}$ $\left[\mathrm{M}^{-1} \mathrm{~cm}^{-1}\right]$ are their molar extinction coefficients. Superscript of the $O D$ denotes the wavelength of photoirradiation, and the subscript is the wavelength of observation. The contents of the parentheses show the time of the photoirradiation. If the sample is continued photoirradiating, the system reaches to a photostationary state, where trans $\rightarrow$ cis and cis $\rightarrow$ trans reactions balance. In the photostationary state, the change of the trans form concentration is cancelled as Eq.6,

$-\frac{d\left(C_{\text {trans }} S l 10^{-3}\right)}{d t}=0$

therefore, using Eq.2, the following equation is obtained after the photostationary state.

$$
\begin{gathered}
\varepsilon_{\text {trans }}(\text { irr }) C_{\text {trans }}^{i r r}(\infty) \phi_{\text {trans } \rightarrow \text { cis }} \\
=\varepsilon_{\text {cis }}(\text { irr }) C_{c i s}^{i r r}(\infty) \phi_{\text {cis } \rightarrow \text { trans }}
\end{gathered}
$$

Here, the ratio of trans/cis concentration under photoirradiation of the wavelength of irr, at photo-stationary state is defined as $R^{i r}$, the ratio of the molar extinction coefficients of trans- to cis-form is defined as $r_{\varepsilon}(\lambda)$, when $\lambda$ is the wavelength of irradiation or observation, and the ratio of the quantum yield of photoreaction of trans $\rightarrow$ cis to cis $\rightarrow$ trans is referred as $r_{\phi}$ in Eqs 8-10.

$$
R^{i r r}=\frac{C_{c i s}^{i r r}(\infty)}{C_{\text {trans }}^{i r}(0)}
$$

$r_{\varepsilon}(\lambda)=\frac{\varepsilon_{c i s}(\lambda)}{\varepsilon_{\text {trans }}(\lambda)}$

$r_{\phi}=\frac{\phi_{\text {cis } \rightarrow \text { trans }}}{\phi_{\text {trans } \rightarrow \text { cis }}}$

Using Eqs 8-10, the Eqs 5 and 7 are simply rewritten in Eqs 11 and 12, respectively.

$$
\frac{O D_{o b s}^{i r r}(\infty)}{O D_{o b s}^{i r}(0)}-1=\left(r_{\varepsilon}(o b s)-1\right) R^{i r r}
$$

$$
r_{\varepsilon}(i r r) \cdot r_{\phi}=\frac{1}{R^{i r r}}-1
$$

Combining Eq. 11 with Eq. 12, Eq. 13 is obtained.

$$
r_{\varepsilon}(i r r) \cdot r_{\phi}=\frac{r_{\varepsilon}(o b s)-1}{\frac{O D_{o b s}^{i r r}(\infty)}{O D_{o b s}^{i r r}(0)}-1}-1
$$

Experimentally, the sample is photoirradiated either at peak wavelength or isosbestic point, and optical density at the "peak" wavelength is observed. Therefore the following four equations are obtained,

$$
\begin{aligned}
& \frac{O D_{\text {peak }}^{\text {peak }}(\infty)}{O D_{\text {peak }}^{\text {peak }}(0)}-1=\left\{r_{\varepsilon}(\text { peak })-1\right\} R^{\text {peak }} \\
& r_{\varepsilon}(\text { peak }) \cdot r_{\phi}=\frac{1}{R^{\text {peak }}}-1 \\
& \frac{O D_{\text {peak }}^{\text {iso }}(\infty)}{O D_{\text {peak }}^{\text {iso }}(0)}-1=\left\{r_{\varepsilon}(\text { peak })-1\right\} R^{\text {iso }} \\
& r_{\phi}=\frac{1}{R^{\text {iso }}}-1
\end{aligned}
$$

where peak and iso denote the wavelength of peak and isosbestic point, respectively. Combining Eqs $14-17$, $r_{\varepsilon}$ (peak) is given in Eq.18.

$$
\left\{r_{\varepsilon}(\text { peak })-\left.\left(\frac{O D_{\text {peak }}^{\text {iso }}(\infty)}{O D_{\text {peak }}^{\text {iso }}(0)}\right)\right|^{\text {peak })-1\}}\left(1+\frac{1}{\left.\frac{O D_{\text {peak }}^{\text {peak }}(\infty)}{O D_{\text {peak }}^{\text {peak }}(0)}-1\right)} \mid\right\}=0\right.
$$

From Eq.18, the ration of the molar extinction coefficient of trans-form to cis-form, at the peak wavelength, $r_{\varepsilon}$ (peak) is obtained in Eq.19.

$$
r_{\varepsilon}(\text { peak })=\left(\frac{O D_{\text {peak }}^{\text {iso }}(\infty)}{O D_{\text {peak }}^{\text {iso }}(0)}\right)\left(1+\frac{1}{\frac{O D_{\text {peak }}^{\text {peak }}(\infty)}{O D_{\text {peak }}^{\text {peak }}(0)}-1}\right)
$$

On the other hand, introducing $r_{\varepsilon}$ (peak) into Eq.14 gives the value of $R^{\text {pedk }}$, and then finally the molar extinction coefficient of cis-form, $\varepsilon_{\text {dis }}\left[\mathrm{M}^{-1} \mathrm{~cm}^{-1}\right]$ at whole wavelength region is calculated from Eq.20. 
$\varepsilon_{c i s}=\left\{\frac{\frac{O D_{\text {peak }}^{\text {peak }}(\infty)}{O D_{\text {peak }}^{\text {peak }}(0)}-1}{R^{\text {peak }}}\right\} \varepsilon_{\text {trans }}$

Fig. 2 presents changes of $O D \mathrm{~s}$ at $317 \mathrm{~nm}$ of azobenzene solutions as the absorption maximum photoirradiated at the same wavelength, and the OD change at $372 \mathrm{~nm}$ as the isosbestic point.

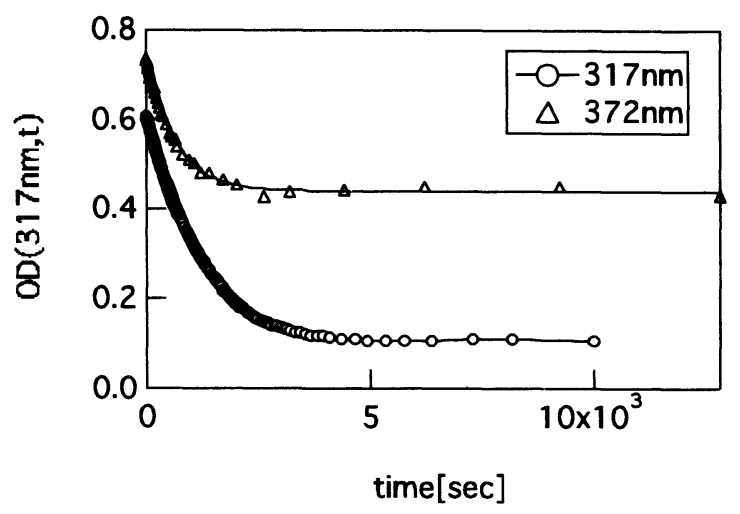

Figure 2. $O D$ changes of $317 \mathrm{~nm}$ of azobenzene in ethanol solutions photoirradiated at of $317 \mathrm{~nm}$ and $372 \mathrm{~nm}$.

The values of $R^{317 m m}$ and $r_{\varepsilon}(317 \mathrm{~nm})$ were 0.903 and 0.084 , respectively. Fig. 3 shows the spectra of $\varepsilon_{\text {cis }}$ and $\varepsilon_{\text {troms }}$ obtained using these values.

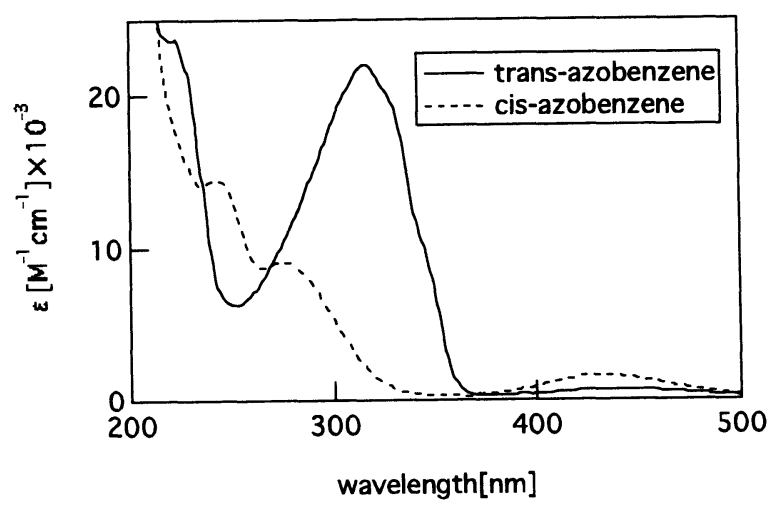

Figure 3. The UV absorption spectra of trans- and cis-azobenzene in ethanol solution.

\subsection{Quantum Yield in Solution}

Fig. 4 shows $O D$ change of azobenzene at $325 \mathrm{~nm}$ during photoirradiation of $325 \mathrm{~mm}$ light. Fitting of the observed $O D$ change to Eq. 3 gives the values of quantum yields for photoreaction of azobenzene, $\phi_{\text {rrans } \rightarrow x i s}$ and $\phi_{c i s-t \text { rmens }}$ as 0.09 and 0.11 , respectively

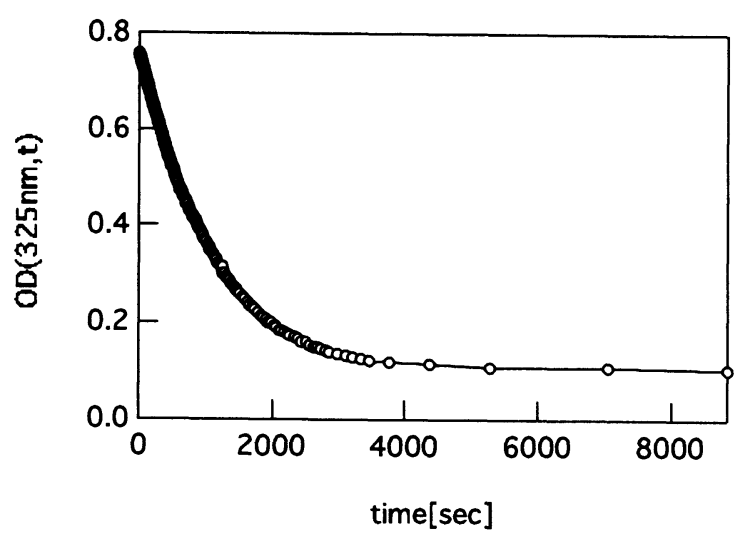

Figure 4. OD change of azobenzene in ethanol solution at $325 \mathrm{~nm}$ during photoirradiation of $325 \mathrm{~nm}$ light $\left(150 \mu \mathrm{W} / \mathrm{cm}^{2}\right)$.

3.3 Photoisomerization of Azobenzene in PMMA Film OD changes of azobenzene in ethanol solution and PMMA film under the same condition are shown in Fig.5. The photoisomerization of azobenzene in ethanol solution follows to the theoretical curve of Eq.3, while the photoisomerization of azobenzene in PMMA solid deviates even in the early stage of the reaction.

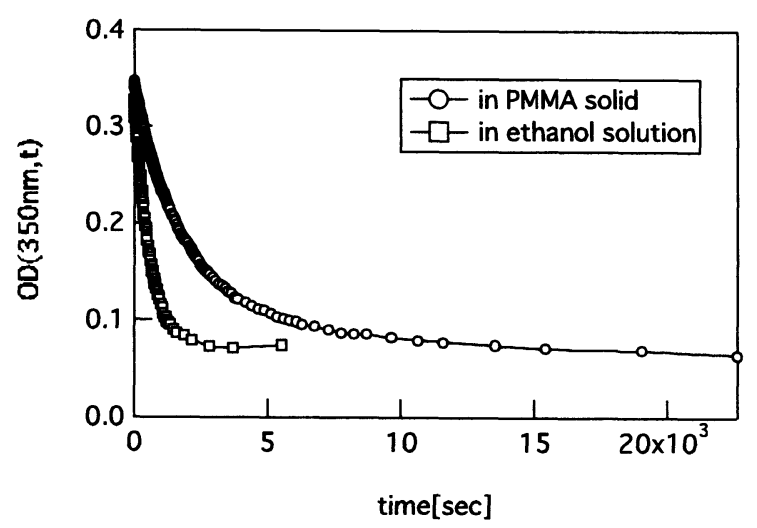

Figure 5. OD changes of azobenzene in ethanol solution and PMMA film during photoirradiation of $350 \mathrm{~nm}$ light under the same condition. Intensity of irradiation light was $270 \mu \mathrm{W} / \mathrm{cm}^{2}$.

This deviation can be attributed to free volume distribution in the polymer solid. The quantum yield of the photoisomerization depends on the size of free volume around the photoreactive molecule. Observed isomerization curve is considered as the sum of curves of various quantum yields. Fig.6 shows the quantum yield distribution of the observed photoisomerization of 


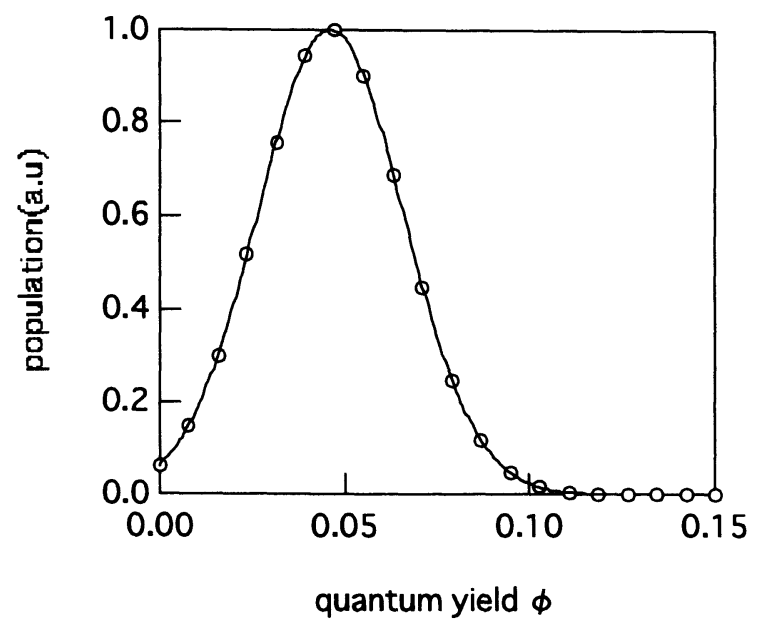

Figure 6. Quantum yields distribution for photoreaction of azobenzene in non-anealed PMMA film.

azobenzene in Fig.5, assuming that the quantum yields have a Gaussian distribution.

The quantum yields range from 0 to 0.11 in Fig.6, which shows the existence of the molecule with quantum yield of zero. Zero indicates that there are the spaces whose free volume size is small than the critical volume for the isomerization in PMMA.

\subsection{Influence of Annealing Temperature}

Fig.7 shows the effect of the annealing on the free volume distribution of the photoisomerization of azobenzene. As annealing temperature went higher, the apparent average of the quantum yield of the photoisomerization decreased. The portion of the molecule with quantum yield of zero also decreased as the temperature increased. The apparent average of the quantum yields and the half width of the quantum yield distribution are listed in Table 1. The narrowing of the distribution of the quantum yield is explained as the molecular relaxation of polymer matrix during thermal curing. The decrease in the portion of the molecule with the quantum yield of zero is also the effect of local relaxation of polymer chains around azobenzene which has been located in the space smaller than the critical free volume.

\section{Conclusion}

We have first demonstrated the analysis of free volume distribution of photochromic probe for the polymer solid state reactions by decomvolution of the observed curve with theoretical expression.

The quantum yield distribution was sensitive to the annealing temperature, which made the distribution narrow. This method is expected to open the door to quantitative understanding of amorphous solid by obtaining distribution of various involved kinetics in the reaction.

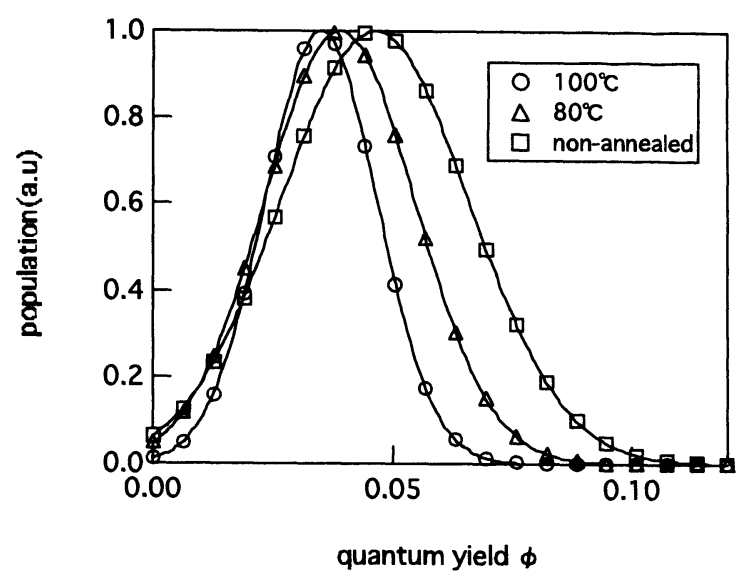

Figure 7. The quantum yields distribution of azobenzene in PMMA films non-annealed and annealed by heating to $80^{\circ} \mathrm{C}$ and $100^{\circ} \mathrm{C}$.

Table 1. Quantum yield and quantum yield distribution of the samples during photoreactions

\begin{tabular}{lcc}
\hline \multicolumn{1}{c}{ Matrix } & Quantum yield & $\mathrm{H}$ \\
\hline PMMA(non-annealed) & 0.05 & 0.046 \\
PMMA(annealing $80^{\circ} \mathrm{C}$ ) & 0.04 & 0.037 \\
PMMA(annealing $100^{\circ} \mathrm{C}$ ) & 0.04 & 0.028 \\
Ethanol & 0.08 & 0.028
\end{tabular}

\section{References}

1. T. Naito, M. Kunishige, T. Yamashita, K. Horie, I. Mita, Reactive Polymers, 15 (1991) 185.

2. M. Asano, F. M. Winnik, T. Yamashita, K.Horie, Macromolecules, 28 (1995) 5861.

3. K. Horie, M. Ikemoto, T. Suzuki, S. Machida, T. Yamashita, N.Murase, Chem. Phys. Lett., 195 (1997) 563.

4. K. Ito, Y. Ujihira, T. Yamashita, K. Horie, $J$. Radioanal. Nuclear. Chem., 210 (1996) 543.

5. H. L. Li, Y. Ujihara, T. Yoshino, T. Tanaka, K. Yashii, T. Yamashita, K. Horie, Polymer, 39 (1998) 4075 .

6. K. Ito, Y. Ujihara, T.Yamashita, K. Horie, J. Polym. Sci. PartB. Polym. Phys., 36 (1998) 1141.

7. T. Yamashita, J. Photopolym. Sci. Technol., 12 (1999) 257. 